\title{
Reflets
}

Revue ontaroise d'intervention sociale et communautaire

\section{Les obstacles à l'intégration au marché du travail des femmes monoparentales à faible revenu}

\section{Pascale Houle}

Volume 9, numéro 2, automne 2003

Travail et mieux-être

URI : https://id.erudit.org/iderudit/011090ar

DOI : https://doi.org/10.7202/011090ar

Aller au sommaire du numéro

Éditeur(s)

Reflets : Revue ontaroise d'intervention sociale et communautaire

ISSN

1203-4576 (imprimé)

1712-8498 (numérique)

Découvrir la revue

Citer cet article

Houle, P. (2003). Les obstacles à l'intégration au marché du travail des femmes monoparentales à faible revenu. Reflets, 9(2), 34-57.

https://doi.org/10.7202/011090ar

\section{Résumé de l'article}

Cette recherche visait à connaître la réalité socio-économique des femmes francophones monoparentales à faible revenu de la ville d'Ottawa et leurs besoins en matière de services en français. Afin d'effectuer la collecte de données, nous avons mis sur pied un groupe de discussion composé de seize femmes francophones. Plusieurs thèmes ont été abordés lors de l'entrevue de groupe, mais, pour les besoins de l'article, seuls certains ont été retenus. En ce qui a trait aux résultats de la recherche, l'analyse montre que le faible niveau d'éducation, l'unilinguisme, le manque de formation et l'inaccessibilité des garderies sont des obstacles rencontrés par les femmes dans leurs démarches d'intégration au marché du travail.
Tous droits réservés (C) Reflets : Revue ontaroise d'intervention sociale et communautaire, 2002
Ce document est protégé par la loi sur le droit d'auteur. L'utilisation des services d’Érudit (y compris la reproduction) est assujettie à sa politique d'utilisation que vous pouvez consulter en ligne.

https://apropos.erudit.org/fr/usagers/politique-dutilisation/ 


\title{
Les obstacles à l'intégration au marché du travail des femmes monoparentales à faible revenu
}

\author{
Pascale Houle ${ }^{1}$ \\ M.S.S., Université d'Ottawa
}

\section{Introduction}

Dans les pays industrialisés, la monoparentalité est un modèle familial en croissance en raison de l'augmentation des unions de fait, des naissances hors mariage et de la fragilité des liens familiaux. De nos jours, les parents et les enfants sont plus nombreux à connaitre un épisode de monoparentalité au courant de leur vie. Cette séquence de vie d'une durée indéterminée rend plusieurs foyers vulnérables sur le plan socio-économique.

Dans la première partie de cet article, nous traiterons des problèmes auxquelles sont confrontées les mères qui sont cheffes ${ }^{2}$ de famille monoparentale en lien avec le marché de l'emploi. La deuxième partie sera consacrée à la méthodologie ainsi qu'à la discussion des résultats d'une recherche effectuée auprès d'un groupe de seize femmes francophones monoparentales à faible revenu. Cette recherche de type exploratoire ${ }^{3}$ avait pour objectif de connaître la réalité socio-économique des femmes francophones monoparentales à faible revenu de la ville d'Ottawa de même que leurs besoins en matière de services en français. Plusieurs thèmes ont été abordés lors de l'entrevue de groupe ${ }^{4}$. Dans cet article, nous n'avons retenu que ceux qui portaient sur les obstacles à l'intégration au marché du travail. 


\section{Problématique}

"Dans le contexte actuel, un emploi à temps complet au salaire minimum n'est pas suffisant pour maintenir une famille au-dessus du seuil de la pauvreté "
L'insertion à l'emploi ne se limite pas uniquement à la volonté de l'individu mais à un ensemble de facteurs. Selon Benveniste et Soleilhavoup (1994), le niveau d'activité d'une cheffe de famille dépend avant tout de son âge, du nombre d'enfants à sa charge et de leur âge. La scolarité de la mère est aussi importante à considérer quant à l'accessibilité des emplois bien rémunérés. Leboeuf (1991) affirme d'ailleurs que la faible scolarité des travailleuses et travailleurs représente une des causes de leur pauvreté.

Actuellement, le marché du travail est plutôt défavorable à l'égard de nombreuses femmes puisqu'il ne leur permet pas d'améliorer leur situation économique. En Ontario, au cours des dernières années, les conservateurs ont mis fin à des lois comme celle de l'équité en matière d'emploi. De même, ils ont réduit certains avantages sociaux au niveau de la sécurité au travail (Table féministe francophone de concertation provinciale de l'Ontario 1998). Il s'avère de plus en plus difficile de trouver un emploi stable et bien rémunéré. Les emplois à temps partiel, temporaires et saisonniers, souvent sans bénéfices, sont de plus en plus fréquents et les emplois à plein temps risquent dans bien des cas d'être mal rémunérés ${ }^{5}$. Dans le contexte actuel, un emploi à temps complet au salaire minimum n'est pas suffisant pour maintenir une famille au-dessus du seuil de la pauvreté (Gupta 2001; Plante 2001).

Par ailleurs, les femmes monoparentales actives sur le marché du travail doivent assumer des rôles multiples. Pourvoyeuse, mère de famille, ménagère, cuisinière, bénévole et étudiante représentent quelques-unes des responsabilités accomplies par les mères seules. Elles assument seules une charge de travail équivalente à celle des familles biparentales. Les cheffes de famille doivent concilier les exigences d'un emploi salarié avec leurs responsabilités familiales (Dandurand et Descarries 1992). La conciliation du travail et de la famille est un défi de taille à relever. Plusieurs obstacles parsèment leur vie quotidienne: le manque de temps, le stress, la fatigue, des heures de sommeil réduites, des loisirs absents ou limités et, parfois, 
le découragement. Certaines femmes optent pour un travail à temps partiel pour se consacrer aux soins des enfants alors que d'autres travaillent à temps plein, cumulant ainsi une surcharge de travail (Leboeuf 1991).

En Ontario, 47 \% des mères monoparentales qui ont des enfants âgés de onze ans et moins sont actives sur le marché du travail salarié (Groupe de travail sur les déterminants de la santé 2000: 8). Plusieurs femmes demeurent ainsi à l'écart du marché de l'emploi et quelques raisons peuvent justifier cette absence: inaccessibilité d'une place subventionnée dans un service de garde, inconvénients occasionnés par le marché du travail comme l'épuisement et manque de temps pour les activités sociales ou récréatives (Sirard, Bélanger, Beauregard, Gagnon, Veillette 1986). En demeurant absentes du marché du travail pendant une période prolongée, certaines femmes, auparavant qualifiées, se trouvent avec des connaissances désuètes ou non reconnues. L'insertion à l'emploi demeure néanmoins le moyen le plus approprié d'éviter une plus grande misère économique (Gunderson et Muszynski 1990). Les femmes sont donc confrontées à faire un choix entre réintégrer un marché du travail inadapté à leur situation familiale ou se contenter d'une aide étatique nettement insuffisante.

\section{La pauvreté chez les femmes cheffes de famille}

Les principales ressources financières des familles monoparentales proviennent des revenus d'emploi, des pensions alimentaires et de l'aide publique (Benveniste et Soleilhavoup 1994). Pour beaucoup d'entre elles, le revenu familial est souvent insuffisant afin de répondre adéquatement aux besoins primaires d'une famille. Selon Statistique Canada, une famille se situe sous le seuil de la pauvreté lorsqu'elle doit consacrer plus de $55 \%$ de son revenu pour se loger, se nourrir et se vêtir (Conseil national du bien-être social 2001). Les familles monoparentales se retrouvent en grand nombre dans cette catégorie. En effet, plus de $52 \%$ des 
"...dans la région

d'Ottawa, les mères monoparentales disposent en moyenne d'un revenu familial inférieur de $51 \% d u$ revenu moyen des familles biparentales habitant la même ville. » cheffes de famille ont vécu dans la pauvreté durant l'année 1998 (Conseil national du bien-être social 2001).

Selon le Conseil national du bien-être social (2001), le revenu familial moyen après impôt d'une femme canadienne cheffe de famille monoparentale en 1998 était de 14954 \$. Plus spécifiquement, dans la région d'Ottawa, les mères monoparentales disposent en moyenne d'un revenu familial inférieur de $51 \%$ du revenu moyen des familles biparentales habitant la même ville ${ }^{6}$. De plus, la différence de revenu la plus significative est celle des hommes par rapport aux femmes. Elles bénéficient d'un revenu moindre (Groupe de travail sur les déterminants de la santé 2000; D'Augerot-Arend, Gauthier, Welch 1996; Dandurand 1995; Leboeuf 1991; Gunderson et Muszynski 1990; Le Gall et Martin 1987). Cette différence de revenu s'explique par un accès à des emplois mieux rémunérés et à l'occupation de postes plus prestigieux chez les hommes que chez les femmes. Par conséquent, les pères chefs de famille se trouvent moins affectés par des difficultés économiques et ont moins de risques de connaitre la précarité. Devault et Bouchard (1996) ont souligné qu'environ $90 \%$ des mères sont préoccupées par l'insécurité financière et par des problèmes économiques alors que ces inquiétudes concernent seulement $46 \%$ des pères ${ }^{7}$.

Dans le cas des mères intégrées au marché du travail, elles doivent répondre aux besoins de leur famille avec un seul salaire souvent peu élevé. Plusieurs femmes vivent en contexte de pauvreté même si elles réintègrent le marché du travail. Elles font face à plusieurs obstacles dont la difficulté à trouver un emploi à temps complet avec un salaire décent et à avoir accès à un service de garde pour les enfants à prix abordable.

\section{Les services de garde pour les enfants}

Selon l'Institut Vanier de la famille (2000), le manque d'accessibilité, le paiement et l'organisation d'un service de garde pour les enfants, s'avèrent problématiques pour un grand nombre de parents 
"Trouver un service de garde à prix abordable représente donc un défi mais encore plus lorsqu'il s'agit d'en trouver un dans sa langue maternelle. " qui travaillent ou étudient à l'extérieur du foyer. Pour les femmes francophones qui résident dans la région d'Ottawa, la situation est compliquée car seulement $11 \%$ des places en garderie sont destinées aux enfants francophones et, parmi celles-ci, $8 \%$ sont subventionnées $^{8}$. Trouver un service de garde à prix abordable représente donc un défi mais encore plus lorsqu'il s'agit d'en trouver un dans sa langue maternelle (D'Augerot-Arend, Gauthier, Welch 1996).

L'obtention d'une place en garderie est un besoin essentiel pour les femmes ayant des enfants en bas âge. Les mères de famille ne peuvent assurer leur présence sur le marché du travail ou étudier si elles ne disposent pas d'un endroit où faire garder leurs enfants. Présentement, les listes d'attente des services de garde s'allongent en raison d'une pénurie de places à coût abordable. En Ontario, le délai d'attente est de plus de deux ans avant de pouvoir bénéficier d'une place subventionnée en garderie ${ }^{9}$. La demande est très élevée mais l'offre demeure très faible. Le nombre de garderies qui ont des places subventionnées est donc insuffisant pour combler les besoins de la population active. Les services de garde au prix du marché restent inaccessibles pour les mères faiblement rémunérées puisqu'elles n'ont pas les capacités financières d'en défrayer leurs coûts. À Ottawa, le tarif mensuel moyen d'un service de garde à l'enfance peut varier entre $637 \$$ et $1141 \$^{10}$. Les femmes qui travaillent à temps plein au salaire minimum devraient verser la presque la totalité de leur salaire afin d'obtenir une place à prix régulier. Le fait de ne pas disposer d'un salaire décent s'avère donc problématique chez les familles monoparentales puisqu'elles doivent payer un service de garde pour les enfants, un logement ainsi que les autres dépenses de la vie quotidienne.

\section{La crise du logement à Ottawa}

Les femmes possèdent peu de marge de manœuvre dans le choix d'un milieu de vie qui convienne à leurs besoins compte tenu de la faiblesse de leur revenu et de la rareté des logements. 
"...les femmes sur

l'assistance sociale pouvaient octroyer entre 60 à $70 \%$ de leurs prestations d'aide sociale an logement. Il ne leur reste plus qu'environ $30 \%$ de leur revenu pour se nourrir, se vêtir et payer les autres dépenses de la vie quotidienne. "
Présentement, la crise du logement à Ottawa aggrave les conditions de vie des familles monoparentales. Les loyers sur le marché privé deviennent de plus en plus inaccessibles en raison de leurs coûts exorbitants. Par ailleurs, le prix moyen des logements locatifs, pour l'année 2000, se situait à 572 \$ pour une garçonnière, à 722 \$ pour un logis comprenant une chambre, à 882 \$ pour un logis comprenant deux chambres et à 1059 \$ pour un logis de trois chambres (Société canadienne d'hypothèques et de logement 2000, cité dans Maxwell 2001).

Selon les Services aux citoyens de la Ville d'Ottawa (2003), le prix des logements a connu une hausse de $25 \%$ depuis 1995 et seuls $5 \%$ de la construction furent des logements locatifs depuis 1996. Les petits salariés et les assistés sociaux doivent consacrer une part importante de leur revenu pour se loger. Au cours de l'année 1996, 41 \% des foyers ont accordé plus de $30 \%$ de leur revenu au logement (Maxwell 2001). Mais, la situation est pire pour les individus qui vivent sous le seuil de la pauvreté comme les assistés sociaux. McAll (1995) a déclaré que les femmes sur l'assistance sociale pouvaient octroyer entre 60 à $70 \%$ de leurs prestations d'aide sociale au logement. Il ne leur reste plus qu'environ $30 \%$ de leur revenu pour se nourrir, se vêtir et payer les autres dépenses de la vie quotidienne. Quelques ménages ont l'opportunité d'accéder à un logement à prix modique. Toutefois, l'offre des logements sociaux ne répond pas à la demande. Les Services aux citoyens de la Ville d'Ottawa (2003) mentionnent que 12500 familles sont en attente d'un logement social. L'accès à ce type de logement demeure restreint puisque le délai d'attente peut être de cinq à huit ans. La situation s'est aggravée surtout depuis le désengagement de l'État qui ne fait qu'augmenter l'acuité de certains problèmes sociaux tel l'itinérance. Au problème du logement s'ajoute une autre contrainte chez les femmes francophones soit celle de ne pas disposer suffisamment de services dans leur langue. 


\section{La spécificité des femmes francophones en milieu minoritaire}

Selon l'Office des affaires francophones (1999), 10,1 \% des familles franco-ontariennes sont monoparentales comparativement à 14,4\% dans l'ensemble de la population en Ontario. Toutefois, entre 1991 et 1996, la monoparentalité chez les familles francophones a augmenté de 9,5\% à 10,1\% (Office des affaires francophones 1999: 6). Le problème linguistique constitue un obstacle supplémentaire à gérer pour les mères francophones seules en Ontario français. De surcroît, la situation des femmes francophones cheffes de famille monoparentale se complique en raison de leur milieu de vie majoritairement anglophone où la disponibilité des services en français est de plus en plus restreinte. Les familles francophones n'ont pas toujours accès à des services dans leur langue. Le manque de professionnels francophones dans la province de l'Ontario a un impact sur la dispensation des services en français (Malenfant et Delisle 1997; D’Augerot-Arend, Gauthier, Welch 1996; Coderre 1995). Selon Kérisit (1997), les femmes immigrantes ont des besoins à combler au niveau de l'accessibilité des services, de la langue, de la formation et de l'emploi. De plus, compte tenu du nombre limité de services en français, les francophones doivent souvent attendre un long moment avant d'être servis dans leur langue (Welch 1995).

Malgré l'adoption de lois visant à protéger les droits linguistiques des francophones, les services en français se font rares et certains organismes disant offrir des services bilingues se trouvent en réalité unilingues anglophones. L'instauration de la Loi 8 sur les services en français de l'Ontario a permis d'assurer aux francophones certains services dans leur langue notamment dans le secteur de la santé et des services sociaux. Toutefois, les municipalités et les institutions non gouvernementales ne sont pas tenues d'appliquer cette loi (Boucher 1994, cité dans Welch 1995). Au niveau de l'éducation, les francophones disposent de très peu de choix en ce qui a trait aux programmes offerts dans la 
langue française. Certains doivent donc choisir leur profession en fonction de la disponibilité des formations (Welch 1995).

Même si les gouvernements ont fourni un effort dans le passé en adoptant la Loi sur les langues officielles qui incita les institutions fédérales à dispenser des services bilingues, les francophones doivent continuer à défendre leurs acquis s'ils veulent protéger leur survie au Canada anglais.

Nous présenterons maintenant quelques éléments de la méthodologie, puis les résultats seront présentés dans la section suivante.

\section{Méthodologie}

L'objectif de la recherche était de connaitre la réalité socioéconomique des femmes francophones monoparentales à faible revenu de la ville d'Ottawa et leurs besoins en matière de services en français. Pour effectuer cette étude, nous avons opté pour un devis de recherche qualitative afin d'explorer le sujet sur le terrain en rencontrant des personnes concernées par la problématique de la pauvreté. De plus, cette recherche exploratoire permettait de brosser un portrait d'une situation spécifique et de favoriser l'émergence d'idées nouvelles (Tripodi, Fellin, Meyer 1985).

Pour pouvoir répondre aux critères d'éligibilité de la recherche, les participantes devaient être des femmes francophones, mères monoparentales, âgées de plus de 18 ans, à faible revenu ${ }^{11}$ et domiciliées dans la ville d'Ottawa. Le groupe devait comporter des femmes de différentes ethnies pour refléter la réalité ethnoculturelle d'Ottawa. Le critère d'être francophone référait à la langue d'usage des participantes.

La promotion et les références furent les deux principales méthodes utilisées pour recruter les participantes de l'échantillon ${ }^{12}$. Afin d'atteindre le nombre requis de participantes, nous avons adopté la technique boule de neige. Plusieurs stratégies ont été utilisées pour recruter les participantes à la recherche mais peu d'entre elles ont donné les résultats escomptés ${ }^{13}$. 
L'entrevue de groupe fut la méthode de cueillette de données privilégiée car elle permet de générer toute une richesse d'informations obtenues par le biais du partage des expériences et du savoir des participantes (Home 1996). Ainsi, nous avons formé un groupe de discussion composé de seize femmes francophones. L'implication demandée consistait à participer à une rencontre d'une durée de deux heures trente qui se tenait dans les locaux d'un organisme communautaire d'Ottawa.

Une fois que les données de notre groupe de discussion ont été compilées, nous avons procédé à une analyse de contenu qui s'est réalisée à partir des étapes développées par Mayer, Ouellet, Saint-Jacques, Turcotte et collaborateurs $(2000)^{14}$. En se référant à l'ouvrage de Landry (1992), nous avons fait appel à une méthode d'analyse inductive fondée sur les similitudes par rapport à la signification des mots exprimés par les répondantes.

Comme toute autre méthode de cueillette de données, l'entrevue de groupe comporte certaines limites. En raison de la taille restreinte de l'échantillon, les résultats de notre étude ne peuvent être généralisables à l'ensemble des femmes francophones cheffes de famille monoparentale à faible revenu de la ville d'Ottawa.

\section{Résultats de la recherche}

Les propos des femmes reflètent l'existence de différents obstacles à l'intégration du marché de l'emploi à Ottawa. Entre autres, la faible rémunération, la discrimination dans l'embauche, la conciliation du travail et de la famille, l'unilinguisme et le manque de formation de langue française sont des barrières mentionnées par les répondantes afin d'expliquer leur exclusion du marché de l'emploi salarié.

En effet, certaines dénoncent que les emplois non spécialisés sont faiblement rémunérés et qu'un seul salaire s'avère insuffisant pour subvenir aux besoins d'une famille. Une d'entre elles 
mentionne recourir occasionnellement au travail au noir dans le but d'augmenter son revenu. Elle croit aussi que les employeurs font parfois de la discrimination dans l'embauche.

Par ailleurs, les résultats suggèrent qu'une grande proportion de femmes trouve difficile d'assumer des rôles multiples et de concilier le travail avec la famille. En analysant les données du groupe de discussion, nous constatons que la monoparentalité semble lourde à supporter pour plusieurs répondantes. Quelquesunes font mention de la charge de travail élevée et des nombreuses responsabilités qu'elles doivent assumer. Selon certaines, le rôle de parent ainsi que l'exécution de toutes les tâches quotidiennes s'avèrent parfois difficile à gérer. Une répondante explique :

Il faut gérer les enfants. Il faut gérer les devoirs d'école. Il faut gérer la maison, la propreté, la nourriture. Il faut gérer toi-même [...]. C'est trop! Moi, je pense que c'est trop pour une personne. Des fois là, moi je n'arrive pas.

L'une d'elles ajoute que le manque de temps pour soi est aussi une réalité.

À Ottawa, en ce qui a trait à l'exigence du bilinguisme dans l'intégration au travail, la collecte des données montre que celle-ci semble un problème plus grand chez les immigrantes que chez les personnes nées au Canada. Plusieurs immigrantes s'expriment en français, sans compter leur langue d'origine, alors que les FrancoOntariennes maitrisent souvent l'anglais et le français. Beaucoup de sentiments de frustration et d'insatisfaction sont exprimés au cours de la discussion en ce qui concerne la réalité des femmes francophones en milieu minoritaire. À plusieurs reprises, nous avons

"...l'intégration au marché du travail dans la capitale nationale est plutôt difficile pour les personnes unilingues francophones. ") pu constater que l'intégration au marché du travail dans la capitale nationale est plutôt difficile pour les personnes unilingues francophones. Les employeurs exigent davantage une main-d'œuvre bilingue compte tenu du milieu majoritairement anglophone. Les exigences linguistiques figurent parmi les qualifications recherchées dans pratiquement tous les domaines d'emplois. D'ailleurs, certaines femmes se questionnent à savoir s'il y a réellement un espace 
pour les francophones sur le marché de l'emploi à Ottawa. L'apprentissage d'une deuxième ou d'une troisième langue constitue un obstacle majeur à surmonter pour celles qui désirent faire partie de la population active. L'obligation d'apprendre l'anglais est dénoncée par un grand nombre de répondantes confrontées à cette réalité depuis leur établissement dans la région. Une femme du groupe affirme: «Les gens qui viennent d'arriver, si vous voulez travailler, faites connaissance de l'anglais, c'est tout. Parce que vous allez être aussi toujours frustrées». Certaines répondantes sans emploi laissent présager que le marché du travail ontarien restera difficilement accessible tant qu'elles ne mấtriseront pas la langue anglaise.

Quelques témoignages font aussi mention de la pénurie de formations en français dans la ville d'Ottawa. La très faible proportion d'établissements offrant une programmation de langue française a pour conséquence de restreindre le choix de professions chez la clientèle francophone. Ainsi, les formations offertes ne conviennent pas nécessairement aux besoins et aux attentes de la population concernée.

D'autre part, le français représente un acquis que l'on doit reconnaitre à sa juste valeur selon certaines répondantes. D'après ces dernières, la maîtrise de la langue française peut augmenter les possibilités d'emploi et ainsi favoriser l'intégration au marché du travail. Une femme du groupe affirme :

On est minoritaire, mais notre français c'est une force! C'est une force qui va nous aider à trouver de l'emploi. [...] Donc, je pense, c'est à nous de pousser, de pousser puisqu'on a une langue. On veut pas que cette langue disparaisse.

Certaines femmes ont encouragé leurs pairs à persévérer et à ne pas perdre espoir malgré les embûches rencontrées. Selon elles, les francophones en ressortiront gagnants à long terme car ils auront la capacité de travailler dans les deux langues officielles.

Pour conclure la question sur l'emploi, nous avons demandé aux femmes d'identifier des solutions et d'expliquer ce qui leur 
conviendrait le mieux au niveau du marché du travail. Leurs réponses furent l'accès à des horaires de travail flexibles, à des emplois à temps partiel et enfin, à un revenu décent.

\section{Vivre au quotidien dans la pauvreté}

Dans le tableau 1 qui suit, on constate que la majorité des femmes qui ont participé à cette recherche sont prestataires d'assistance sociale. Celles qui occupent un emploi ne disposent pas d'un revenu assez élevé leur permettant de se situer au-dessus du seuil de la pauvreté. De surcroit, toutes les travailleuses se caractérisent par une précarité d'emploi puisqu'elles indiquent que leur travail est soit temporaire ou contractuel. Ainsi, ces femmes qui vivent en contexte de pauvreté rencontrent des problèmes financiers importants étant donné leur faible revenu.

Tableau 1 - Profil socio-économique des répondantes

\begin{tabular}{llcc} 
& \multicolumn{1}{c}{ Caractéristiques } & $\begin{array}{c}\text { Participantes } \\
\mathrm{N}=16\end{array}$ & $\begin{array}{c}\text { Pas de } \\
\text { réponse }\end{array}$ \\
\hline \hline Âge & Moins de 35 ans & 7 & 1 \\
& 35 ans et plus & 8 & 1 \\
\hline État civil & Séparée ou divorcée & 11 & \\
& Célibataire & 3 & 0 \\
& Veuve & 1 & 1 \\
\hline Nombre d'enfants & 1 enfant & 5 & \\
à charge (N=32) & 2 enfants & 7 & \\
& 3 enfants et plus & 4 & \\
\hline Âge des enfants & Moins de 5 ans & 3 & \\
& 5 à 11 ans & 15 & 13 \\
\hline Domicile & 12 ans et plus & 16 & \\
& Locataire & 0 & \\
\hline
\end{tabular}


Tableau 1 - Profil socio-économique des répondantes (suite)

\begin{tabular}{llcc} 
& \multicolumn{1}{c}{ Caractéristiques } & $\begin{array}{c}\text { Participantes } \\
\text { N }=16\end{array}$ & $\begin{array}{c}\text { Pas de } \\
\text { réponse }\end{array}$ \\
\hline \hline Type de logis & Logis non subventionné & 7 & 2 \\
& Logis subventionné (HLM, Coop) & 6 & \\
& Autres & 1 & 1 \\
\hline Type d'activités & Études ou formation & 7 & 0 \\
& En emploi & 5 & 0 \\
\hline Marché du travail & Bénévolat & 4 & 0 \\
& En emploi & 5 & \\
\hline Type d'emploi & Sans emploi & 11 & \\
\hline Nombre d'heures de & Temporaire ou contractuel & 5 & \\
travail par semaine & Moins de 25 heures & 2 & \\
\hline Source de revenu & Plus de 25 heures & 3 & \\
& Revenu d'emploi & 4 & \\
\hline Revenu annuel & Assistance sociale & 11 & \\
& Autres & 1 & \\
& $5000 \$$ à $9000 \$$ & 5 & 5 \\
& $10000 \$$ à $14000 \$$ & 2 & 2 \\
\hline \hline
\end{tabular}

L'analyse des entrevues confirme que le manque d'argent concerne la majorité des répondantes. Plusieurs expliquent que les difficultés surviennent après le paiement du loyer car il reste très peu d'argent pour défrayer les coûts reliés au téléphone, à la nourriture et aux autres dépenses. Quelques femmes affirment ne pas disposer des ressources financières nécessaires leur permettant d'offrir un camp d'été ou un service de gardiennage à leurs enfants. Même en bénéficiant d'une subvention, il s'avère parfois difficile de fournir une contribution financière pour les mères monoparentales vivant dans une pauvreté extrême. Tout compte fait, les résultats de la recherche montrent que les barèmes de l'assistance sociale sont trop bas pour subvenir aux besoins d'une famille. Certaines femmes qui ont fait le calcul de leurs 
revenus et dépenses se voient dans l'obligation de se priver au niveau de la nourriture. Une répondante affirme:

Mon «rent» est 600.100\$ par mois à l'hiver là. Ça fait 700 plus mon téléphone, plus mon Sprint Canada (Basic Cable), ça fait $740 \$ .760 \$$ avec mon assurance. Pis mon chèque est de 840-844\$ tsé.Y reste $144 \$$ à manger par mois.

"Une autre femme $d u$ groupe présente un bilan de sa situation financière et souligne qu'elle se trouve parfois dans l'incapacité de donner une collation à son enfant lorsqu'il va à l'école. "
Une autre femme du groupe présente un bilan de sa situation financière et souligne qu'elle se trouve parfois dans l'incapacité de donner une collation à son enfant lorsqu'il va à l'école. Elle se sent impuissante puisqu'elle n'a pas la capacité financière d'acheter suffisamment de nourriture. Elle explique:

L'aide sociale me donne 849 \$ et je paie un petit appartement d'une chambre à $700 \$$. [...] Je paie mon téléphone une cinquantaine de dollars et pis l'enfant n'a pas de distraction s'il n'a pas un câble plutôt pour la télévision qui me coûte une quarantaine de dollars. Et moi-même, je dois me payer des médicaments chaque mois. L'enfant doit partir à l'école avec une collation [...]. Des fois, je ne donne pas la collation à l'enfant mais à son retour j'ai des notes de l'école. [...] J'essaie d'expliquer à l'école mais en vain, ils ne comprennent pas. Pour eux, l'enfant doit se présenter avec une collation.

Plusieurs femmes monoparentales se voient maintenues dans une situation de pauvreté. Ces familles se voient donc prises dans un cercle vicieux auquel il s'avère difficile d'échapper.

\section{L'inaccessibilité des services de garde}

En ce qui a trait au gardiennage, les résultats indiquent de longues listes d'attente pour les places subventionnées, le manque de 
"L'inaccessibilité des services de garde constitue un obstacle important pour les femmes qui désirent suivre une formation ou intégrer le marché du travail afin d'échapper à la pauvreté. " flexibilité au niveau des critères d'éligibilité donnant droit à la subvention, une pénurie de gardiennes, un problème d'accès pour les enfants âgés de dix ans et plus et le prix excessivement élevé des garderies sur le marché privé. Une répondante affirme: «Pour un enfant à temps plein, avant et après l'école, ça peut coûter 75 \$ pour un enfant. Donc, j'en ai deux. Donc, 150 \$ par semaine, 600 \$ par mois ».

Une autre participante évoque les listes d'attente et les critères d'éligibilité pour les places subventionnées. Elle explique:

T'as pas l'argent pour payer pour une gardienne. Té sur une liste d'attente pour une subvention. C'est pas évident non plus d'avoir la subvention pis si ton enfant est au-dessus l'âge de la subvention, tu peux pas avoir de gardienne pour cet enfant là. C'est ça ma situation. Comme moi, j'entreprends d'aller à l'Université en septembre pis j'ai pas les sous pour payer pour une gardienne surtout pas pour deux enfants. Donc, je me retrouve dans une situation qui n'est pas facile.

L'inaccessibilité des services de garde constitue un obstacle important pour les femmes qui désirent suivre une formation ou intégrer le marché du travail afin d'échapper à la pauvreté. Les familles monoparentales à faible revenu ne peuvent se permettre de payer un service de garde au prix régulier car une grande partie de leur revenu est consacrée au paiement du logis.

\section{Le logement au cœur de leurs préoccupations}

Lorsque nous demandons aux participantes de la recherche de décrire leur réalité actuelle au niveau du logement, une grande proportion rapporte des situations problématiques qu'elles ont vécues ou dans lesquelles elles se retrouvent présentement en tant que mère cheffe de famille monoparentale. Entre autres, la majorité des répondantes dénoncent des conditions de logement 
"Compte tenu de la crise du logement et du prix excessivement élevé des loyers, les femmes se considèrent très limitées dans le choix de leur logis." inacceptables telles que l'insalubrité, le manque d'espace, la négligence du propriétaire, la peur d'être évincée et le prix inabordable des loyers. En ce qui concerne l'état des logements, certaines mentionnent les problèmes de moisissure, de plomberie, de bruits ou de présence d'insectes et du manque d'insonorisation. Compte tenu de la crise du logement et du prix excessivement élevé des loyers, les femmes se considèrent très limitées dans le choix de leur logis. De plus, les propriétaires exigent parfois un endosseur aux locataires afin de s'assurer du paiement. Les femmes valorisent les logements sociaux étant donné leur prix abordable. Cependant, elles dénoncent leur manque d'accessibilité et les critères de priorité trop rigides.

Plusieurs femmes affirment que les logements sociaux affichent de longues listes d'attente. Selon leurs témoignages, le délai d'attente peut varier entre six mois et huit ans. Certaines trouvent que cette réalité n'est pas adaptée aux besoins immédiats des familles qui vivent dans une précarité financière. Une femme mentionne :

La plupart du monde prend des logements à prix modique mais la liste d'attente est de sept à huit ans. Dans sept à huit ans, j'vas avoir faite mon université, mon p'tit va avoir dix ans. J'en aurai pu besoin! Y a pas de logique là! [...].

Le processus semble très long même pour les familles sans logis. Une répondante affirme avoir vécu avec ses enfants dans un abri d'urgence pendant une période approximative d'un an. Selon elle, il s'agissait de la seule procédure à suivre pour pouvoir accéder à un logement subventionné. Ses conditions d'habitation restent peu satisfaisantes puisqu'elle a dû accepter un logement qui ne correspondait pas à ses attentes. D'autre part, la présence de problèmes de santé n'a pas accéléré le traitement de la demande d'une des femmes du groupe. La priorité lui a été refusée car son état de santé ne démontrait pas une gravité suffisamment importante. Certes, un grand nombre de femmes déclarent vivre des situations injustes et certaines subissent même de la discrimination. 
"Les résultats de la recherche démontrent que le paiement $d u$ loyer constitue une source d'angoisse majeure pour plusieurs répondantes. "
Dans la recherche de logement, certaines disent être discriminées par rapport à leur statut économique. Ainsi, lorsqu'elles mentionnent l'assistance sociale comme source de revenu, il semble plus difficile d'obtenir le logement souhaité. Une répondante ajoute que la présence d'enfants peut aussi faire l'objet de discrimination: "C'est difficile souvent, un appartement quand t'as deux enfants, t'ai toute seule pis t'ai sur le "welfare». C'est simple. T'as des enfants. Oh non! On veut pas avoir d'enfants ici. C'est contre la loi de dire ça».

D'autres femmes expriment avoir vécu de la discrimination par rapport à leur ethnie ou à la couleur de leur peau. Ces actes discriminatoires proviennent dans certains cas des propriétaires ou des locataires habitant le même immeuble.

Les résultats de la recherche démontrent que le paiement du loyer constitue une source d'angoisse majeure pour plusieurs répondantes. Cette dure réalité affecte particulièrement les femmes dont la principale source de revenu provient de l'assistance sociale et les travailleuses à faible revenu qui occupent un logement sur le marché privé. La crainte de manquer de ressources financières ou de se faire évincer de son appartement occasionne des sentiments de stress et d'insécurité qui peuvent nuire au bienêtre des enfants. Une femme indique:

Même si on travaille, qu'on est pas sur l'aide sociale, on a cette tension. Quand on a un loyer, qu'on est locataire privé. À chaque mois, tu dois payer son mois. [...] Alors, ça c'est vraiment une tension. Ça n'aide pas moralement. Si on est sous le stress, les enfants souffrent!

En plus de la part élevée du revenu consacré au paiement du logement, il faut ajouter les coûts de l'électricité et du chauffage. L'une d'entre elles qui habite dans un logement subventionné affirme que le coût mensuel de l'électricité est plus dispendieux que son loyer. De plus, elle ajoute que ses prestations d'assistance sociale ont diminué en conséquence de la baisse du prix de son logis. D'autres redoutent les coupures d'Hydro en cas d'incapacités 
financières. Pour la plupart des répondantes, presque tout le revenu est réservé au paiement du loyer ainsi qu'aux comptes s'y rattachant. Une femme évoque: «J'habite dans un logement privé. Je paie 935.00 \$. L'électricité et chauffage n'est pas compris là dedans mais quand j'ai fini de payer la maison, de l'argent, y en a plus».

\section{Discussion}

\section{"Nous constatons aussi que toutes les participantes de la recherche intégrées au marché du travail occupent un emploi précaire. "}

Les résultats de la recherche suggèrent que la monoparentalité peut devenir lourde à supporter, car en étant l'unique soutien de la famille, les femmes doivent prendre en charge toutes les tâches s'y rattachant. La charge élevée de travail, les nombreuses responsabilités à accomplir, le rôle de parent à assumer seule, constituent le lot des familles monoparentales. La difficile conciliation du travail et de la famille, qui ressort de notre analyse, rejoint l'étude de Dandurand et Descarries (1992). L'accomplissement de rôles multiples peut devenir difficile à gérer particulièrement pour les mères seules.

Par conséquent, même si l'intégration au marché de l'emploi peut enrayer la pauvreté, elle ne garantit pas de meilleures conditions économiques. Nos données suggèrent que les emplois exigeant peu de qualifications sont faiblement rémunérés et qu'un seul salaire peu élevé demeure nettement insuffisant pour répondre aux besoins d'une famille monoparentale. Selon Gupta (2001) et Plante (2001), un emploi à temps plein au salaire minimum ne permet pas à une famille de sortir de la pauvreté. Nous constatons aussi que toutes les participantes de la recherche intégrées au marché du travail occupent un emploi précaire. Cela s'explique en partie par l'augmentation des emplois à temps partiel, temporaires et saisonniers (Campagne 2000, cité dans Groupe de travail sur les déterminants de la santé 2000). Les problèmes économiques rencontrés par les femmes monoparentales vont dans la même direction que les écrits de plusieurs auteurs ${ }^{15}$. Devault et Bouchard (1996) ajoutent qu'approximativement $90 \%$ des 
"...il existe une

pénurie de formations de langue française dans la ville

d'Ottawa. " femmes monoparentales manifestent des inquiétudes sur le plan financier.

Au niveau de l'éducation, les données révèlent qu'il existe une pénurie de formations de langue française dans la ville d'Ottawa. Les francophones doivent déterminer leur choix de profession en fonction des champs d'étude disponibles dans leur langue (Welch 1995) ou s'orienter vers les formations de langue anglaise. Nous avons aussi observé que l'exigence de l'anglais est particulièrement problématique chez les femmes immigrantes d'expression française. Or, plusieurs répondantes ont identifié des barrières linguistiques au niveau du marché du travail et de la formation offerte. Ces résultats s'apparentent à la réflexion de Kérisit (1997) sur les besoins des femmes immigrantes en matière de formation, d'emploi, de langue et de services.

Dans une autre perspective, il est intéressant de constater que le français en milieu minoritaire est perçu comme un acquis important à préserver. Comme le mentionnent certaines répondantes, la maîtrise de la langue française constitue une force puisqu'elle est une qualification recherchée par les employeurs.

Par rapport au gardiennage, les résultats démontrent des longues listes d'attente, des difficultés d'accès, un manque de gardiennes et des coûts excessivement élevés. Ces observations confirment les écrits consultés (Institut Vanier de la famille 2000; Région d'Ottawa-Carleton 1998, cité dans Groupe de travail sur les déterminants de la santé 2000).

D'autre part, la recherche de logement est problématique chez certaines femmes du groupe compte tenu de la pénurie de logements occasionnée en partie par le désengagement des gouvernements dans la construction de logements sociaux (Conseil canadien de développement social 1998; cité dans Groupe de travail sur les déterminants de la santé 2000). Les femmes qui ont participé à notre recherche sont confrontées à un choix de logements très restreint en raison de leur situation économique et du taux d'inoccupation extrêmement bas. D'après les Services aux citoyens de la ville d'Ottawa (2003), le coût des loyers a augmenté de $25 \%$ en 1995 et seuls $5 \%$ des nouvelles constructions étaient réservés à des logements locatifs. Il n'est donc pas surprenant 
que les femmes se voient dans l'obligation d'habiter dans des appartements insalubres et inadéquats. À cet effet, rappelons que le bruit, l'insalubrité, le manque d'espace, la négligence du propriétaire, la présence d'insectes sont, entre autres, les problèmes soulevés par répondantes. De même, les résultats indiquent qu'il existe une discrimination fondée sur la situation financière, la présence d'enfants, l'ethnie et la couleur de la peau des répondantes. Des écrits, allant dans la même direction, mentionnent que des femmes cheffes de famille auraient été victimes de discrimination par les propriétaires et les concierges (Escomel et Lord 1990; cité dans Dandurand 1995). Notre analyse soulève aussi le manque d'accès aux logements sociaux. En effet, les longues listes d'attente confirment que l'offre ne répond pas à la demande. On estime que 12500 familles étaient inscrites au registre des logements sociaux au début de l'année 2003 (Services aux citoyens de laVille d'Ottawa 2003). Certaines participantes à la recherche ont dû habiter dans des abris d'urgence avant de pouvoir accéder à un logement social. Certes, le paiement du loyer rend beaucoup de femmes anxieuses particulièrement celles qui vivent de l'assistance sociale. Comme nous l'avons observé, la presque totalité du revenu des familles, vivant sous le seuil de la pauvreté, est consacrée au logement. Ces dernières doivent donc composer avec moins que le minimum nécessaire pour répondre à leurs besoins essentiels.

\section{Conclusion}

De nombreuses familles monoparentales dirigées par une femme vivent dans une situation de pauvreté et celle-ci les oblige à vivre dans des conditions de survie. Suite à une rupture conjugale, certaines femmes deviennent dépendantes de l'assistance sociale ou encore, elles intègrent un marché du travail qui n'a pas de meilleures conditions de vie à leur offrir. La précarité d'emploi maintient plusieurs familles dans une pauvreté à laquelle il s'avère difficile d'échapper. Par ailleurs, différents obstacles limitent 
l'intégration des femmes sur le marché du travail. Les résultats de la recherche démontrent que l'inaccessibilité des services de garde, la faible rémunération, la discrimination dans l'embauche, la conciliation du travail et de la famille, l'unilinguisme ainsi que le manque de formation de langue française sont quelques-uns des facteurs qui expliquent en partie l'exclusion des femmes du marché de l'emploi salarié. Afin de pouvoir surmonter ces obstacles, les femmes monoparentales ont besoin de certaines conditions minimales leur permettant de participer au développement de la collectivité et d'acquérir une autonomie financière. Par contre, nous sommes conscients que le vécu des femmes est parsemé d'embûches et que dans bien des cas, il ne s'agit pas d'une question de volonté mais bien de possibilités. Comme le mentionne Lévesque:

Pour une mère seule, élever des enfants relève de l'héroïsme: réussir à arriver malgré la pauvreté, être mère et tenter d'occuper un emploi, faire une course aux diplômes malgré les responsabilités familiales assumées seules et maintenir sa stabilité émotive malgré la fatigue (Lévesque 2002: 46).

Pour terminer, nous croyons qu'il serait essentiel que des recherches s'intéressent davantage à la réalité des femmes monoparentales afin d'améliorer leur qualité de vie. Nous souhaitons aussi que cet article puisse contribuer à l'avancement des connaissances sur les obstacles à l'intégration au marché du travail des femmes monoparentales à faible revenu et incite les chercheuses et chercheurs, les praticiennes et praticiens ainsi que les citoyennes et citoyens à participer collectivement à la recherche de solutions qui collent davantage à leur réalité.

\section{Bibliographie}

BENVENISTE, Corinne et Jeanine SOLEILHAVOUP (1994). Les familles monoparentales: portrait social, Paris, INSEE.

CANADIAN COUNCIL ON SOCIAL DEVELOPMENT (2003). «Statistics Canada’s Low Income Cut-Offs», The Daily, February 5, 2003. 
CODERRE, Cécile (1995). «Femmes et santé, en français s'il vous plaît», Reflets, vol. 1, no 2, 38-71.

CONSEIL NATIONAL DU BIEN-ÊTRE SOCIAL (2001). Profil de la pauvreté infantile, 1998, Ottawa, Ministre des Travaux publics et services gouvernementaux Canada.

DANDURAND, Renée B. (1995). Divorce et nouvelle monoparentalité, dans Fernand Dumont, Simon Langlois et Yves Martin, éd., Traité des problèmes sociaux, Québec, IQRC, 519-544.

DANDURAND, Renée B. et Francine DESCARRIES (1992). Mères et travailleuses: de l'exception à la règle, Québec, Institut québécois de recherche sur la culture.

D'AUGEROT-AREND, Sylvie, GAUTHIER, Lise et David WELCH (1996). Femmes francophones de la région torontoise face aux lois et aux services en matière de séparation, de divorce et $d u$ bien-être des enfants: rapport final, Toronto, Conseil de recherche en sciences humaines du Canada.

DEVAULT, Annie et Camil BOUCHARD (1996). «Difficultés vécues et stratégies de résolution de problèmes: une comparaison entre pères et mères de familles monoparentales», Revue québécoise de psychologie, vol. 17, no 3, 5-23.

GROUPE DETRAVAIL SUR LES DÉTERMINANTS DE LA SANTÉ (2000). Les défis auxquels font face nos enfants: bulletin sur la pauvreté des enfants à Ottawa-Carleton, Ottawa, Conseil de Planification sociale d'Ottawa-Carleton.

GUNDERSON, Morley et Leon MUSZYNSKI (1990). Vivre ou survivre? Les femmes, le travail et la pauvreté, Ottawa, Conseil consultatif canadien sur la situation de la femme.

GUPTA, Meenakshi (2001). «Primes et licenciements: une histoire de deux phénomènes constatés dans la population active», dans Notre Capitale Sociale, vol. 1, no 1, 5-9.

HOME, Alice (1996). «Enhancing research usefulness with adapted focus groups», Groupwork, vol. 9 , no 2, 128-138.

KÉRISIT, Michèle (1997). «Immigrantes et réfugiées francophones en Ontario: quelques pistes de réflexion", dans Linda Cardinal, éd., À partir de notre expérience. Femmes de la francophonie ontarienne, Cahiers réseau de recherches féministes, no 4, Québec, Université du Québec à Montréal, Institut de recherches féministes, 75-89.

KÉRISIT, Michèle et Nérée ST-AMAND (1997). «Résistance et créativité: pratiques alternatives des familles démunies», Reflets, vol. 3, no 1,30-53.

LANDRY, Réjean (1992). «L'analyse de contenu», dans Recherche sociale: de la problématique à la collecte des données, Benoît Gauthier (éd.), Sainte-Foy, Presses de l’Université du Québec, 337-359.

LEBOEUF, Louise (1991). «Les femmes et la pauvreté», Service social, vol. 40, no 3, 24-41.

LE GALL, Didier et Claude MARTIN (1987). Les familles monoparentales: évolution et traitement social, Paris, Les Éditions ESF.

LÉVESQUE, Sylvie (2002). «La séparation conjugale: un gain ou une perte pour les femmes?», dans Séparation conjugale: pour qui les gains, pour qui les pertes? Actes du colloque de recherche tenu dans le cadre du $70^{\circ}$ Congrès de l'ACFAS, Québec, Centre de recherche sur l'adaptation des jeunes et des familles à risque, Université Laval, 43-46.

L'INSTITUTVANIER DE LA FAMILLE (2000). Profil des familles canadiennes II, Ottawa, l'Institut Vanier de la famille.

MALENFANT, Brigitte et Louise DELISLE (1997). «Besoins des francophones à l'égard des services communautaires d'Ottawa-Carleton", Reflets, vol. 3, no 1, 143-148.

MAXWELL, Timothy (2001). «Le logement à Ottawa», Notre capitale sociale, vol. 1, no 2, 6-7. 
MAYER, Robert, Francine OUELLET, Marie-Christine SAINT-JACQUES, DanielTURCOTTE et al. (2000). Méthodes de recherche en intervention sociale, Boucherville, Gaëtan Morin éditeur.

MCALL, Christopher (1995). «Le cercle vicieux de l'aide sociale», Policy options politiques, mai, 2932.

OFFICE DES AFFAIRES FRANCOPHONES (1999). Les femmes francophones en Ontario: profil statistique, Toronto, Gouvernement de l'Ontario.

PLANTE, Nathalie (2001). «Soutien communautaire à l'emploi», Notre Capitale Sociale, vol. 1, no 1, $13-15$.

SERVICES AUX CITOYENS,VILLE D'OTTAWA (2003). Leadership en logement abordable à Ottawa, présentation de Jocelyne St-Jean à l'Assemblée générale annuelle d'Action-Logement, 31 mars 2003, Ottawa, document ronéotypé.

SIRARD, Guylaine, BÉLANGER, Francine, BEAUREGARD, Céline, GAGNON, Sylvie et Denis VEILLETTE (1986). Des mères seules: une étude sur la situation des femmes cheffes de famille monoparentale du Centre-Sud de Montréal, Montréal, Les entreprises Berga Inc.

TABLE FÉMINISTE FRANCOPHONE DE CONCERTATION PROVINCIALE DE L'ONTARIO (1998). Le travail obligatoire en Ontario: solution ou imposture?, réalisation d'Angèle Gagnon, 20 min, Ottawa.

TRIPODI, Tony, Philip FELLIN et Henry MEYER (1985). «Vue d'ensemble du système de classification", dans The Assessment of Social Research, extraits des pages 16-47 et 66-77, synthèse des chapitres 2 et 4 préparée par Alice Home (1997), Itasca, Peacock.

WELCH, David (1995). «Les Franco-Ontariens: la résistance comme mode de vie», Reflets, vol. 1, no $1,20-42$.

\section{Notes}

1. Pascale Houle a reçu deux bourses dont celle de Roland Lecomte de l'École de service social et la bourse Monseigneur Joseph-Aurèle Plourde 2002 de la faculté des Études Supérieures de l'Université d'Ottawa. La première bourse vise à promouvoir la recherche sur les communautés francophones et la seconde est attribuée à un projet de recherche qui tend à rehausser la qualité de vie des personnes défavorisées, de préférence au sein de la communauté locale.

2. L'utilisation du terme féminisé "cheffes» est le choix de l'auteure de l'article (NDRL).

3. Cette recherche a été effectuée dans le cadre d'un mémoire de maîtrise en service social. Houle, Pascale (2003), «Mères seules pauvres: entre le vouloir et le pouvoir». Le point de vue des femmes francophones monoparentales à faible revenu de la ville d'Ottawa sur leurs besoins et les services en français dans la communauté», Ottawa, Université d'Ottawa, École de service social.

4. Les thèmes qui ont été traités lors de l'entrevue de groupe sont: le logement, le marché du travail, le revenu familial, les services de garde, les pensions alimentaires pour enfants, les spécificités des femmes francophones en milieu minoritaire, les spécificités des femmes immigrantes et le réseau social.

5. Campagne 2000, cité dans Groupe de travail sur les déterminants de la santé 2000.

6. Statistique Canada s.d., cité dans Groupe de travail sur les déterminants de la santé 2000:5.

7. Voir l'article de Devault et Bouchard 1996: 13. 
8. Regroupement des services de garde de langue française d'Ottawa-Carleton 1998, cité dans Groupe de travail sur les déterminants de la santé 2000: 15.

9. Région d'Ottawa-Carleton 1998, cité dans Groupe de travail sur les déterminants de la santé 2000.

10. Région d'Ottawa-Carleton 1998, cité dans Groupe de travail sur les déterminants de la santé 2000 .

11. Les seuils de faible revenu ont été établis par Statistiques Canada, pour l'année 2002 (Canadian Council On Social Development 2003; «Statistics Canada’s Low Income Cut-Offs», The Daily, February 5, 2003.

12. Des intervenantes et intervenants ont présenté le projet de recherche et transmis nos coordonnées aux femmes qui correspondaient aux critères d'éligibilité. Les personnes intéressées ont communiqué avec nous par téléphone de leur propre gré pour confirmer leur présence à la rencontre.

13. Des affiches de recrutement ont été distribuées et affichées sur les babillards d'un grand nombre d'organismes communautaires et d'une université à Ottawa. Des présentations orales ont été effectuées dans des comités et des assemblées. L'envoi de courriels et de télécopies a fait partie des outils de communication utilisés. Un réseau francophone a diffusé l'information accompagnée de l'affiche de recrutement à une liste d'intervenants via l'Internet. Les stratégies les plus utiles ont été les appels téléphoniques effectués à des intervenantes et intervenants d'organismes communautaires de première ligne ainsi que la remise d'une compensation financière aux participantes à la recherche.

14. Suite à la transcription de l'enregistrement de la discussion de groupe, nous avons fait une pré analyse des données en effectuant une lecture flottante. Celle-ci a permis de codifier les données et de définir les catégories d'analyses. Un guide de codification a été conçu afin de repérer toutes les observations qui correspondaient à l'unité d'analyse sélectionnée. L'analyse et l'interprétation des résultats ont été réalisées puis elles ont été soumises à des fins de vérification.

15. Voir à ce sujet: Conseil national du bien-être social 2001; Kérisit et St-Amand 1997; Devault et Bouchard 1996; Gunderson et Muszynski 1990. 